\title{
Comparison of Amplitude Variations of Seasonal and Sub-Seasonal Oscillations in Length of Day and Total Solar Irradiance
}

\author{
Wiesław Kosek', Justyna Gabryszuk ${ }^{2 *}$ \\ 1 Faculty of Civil Engineering and Geodesy, Military University of Technology, ul. gen. Sylwestra Kaliskiego 2, \\ 00-908 Warsaw, Poland \\ 2 Faculty of Production Engineering, University of Life Sciences in Lublin, ul. Akademicka 13, 20-950 Lublin, \\ Poland \\ * Corresponding author's e-mail: justyna.gabryszuk@up.lublin.pl
}

\begin{abstract}
The aim of the study was to identify and compute oscillations in two different time series with similar amplitude variations using length of day data with tide model removed (LODR) and total solar irradiance (TSI) data. The combination of the Fourier transform band pass filter and Hilbert transform allows detecting amplitude variations as a function of the oscillation period. The amplitude variations in two different time series enable computation of frequency dependent or time-frequency correlation coefficients between them. It allows also identifying such oscillations in two time series which have similar amplitude variations. The method applied to LODR and TSI data, enable to detect a possible relationship between them. This comparison method can be applied to any time series which consist of oscillations with non-constant amplitudes.
\end{abstract}

Keywords: length of day, El Nino, solar irradiance, Fourier transform, Hilbert transform

\section{INTRODUCTION}

The modern satellite and space geodetic techniques such as Global Navigation Satellite Systems (GNSS), Satellite Laser Ranging (SLR), Lunar Laser Ranging (LLR) Very Long Baseline radio - Interferometry (VLBI), Doppler Orbitography and Radiopositioning Integrated by Satellite (DORIS) enable accurate determination of the Earth orientation parameters (EOP) with a precision corresponding to few millimeters on the Earth's surface. EOP consist of $x$, y pole coordinates, universal time UT1-UTC and $\mathrm{dX}, \mathrm{dY}$ precession-nutation corrections and are provided by the International Earth Rotation and Reference System Service (IERS). Their forecasts are used for real-time transformations between the international celestial and terrestrial reference frames for the positioning of objects in space outside the rotating Earth [Gambis 2004; Kalarus et al. 2010].

Length of day (LOD) is the first derivative of UT1-TAI which is UT1-UTC with leap seconds removed. It describes the irregular rotation of the earth around its axis, due to different geophysical reasons, mainly caused by solid Earth and ocean tides [Ray and Erofeeva 2014; Yoder et al. 1981] as well as by the exchange of angular momentum between solid Earth and atmosphere at seasonal and subseasonal time scales [Kosek 1993; Lei et al. 2015; Zheng et al. 2003]. Longer period variations ranging from about 2 to several years in LOD are associated with the ENSO (El Niño Southern Oscillation) phenomenon [Chao 1989; Dickey et al. 1992; Dickey et al. 1994; Eubanks et al. 1986; Gambis 1992; Haddad et al. 2017; Liu et al. 2005]. The decadal LOD fluctuations are caused by the exchanges of angular momentum between the core and mantle [Gross 2007; Jault and Le Mouël 1991]. Non-tidal variations of LOD are named length of day residuals (LODR). The heating of different parts of the Earth's surface with the annual period caused by the Earth's orbital motion induces the thermal annual cycle in the Earth's fluid layers. The basic source that 
causes the exchange of angular momentum between solid Earth and atmosphere as well as the source of excitation of the ENSO phenomenon may be solar activity. The relationship between solar activity and the variable rotation of the Earth has been the subject of research in the past. Langley et al. [1981] found a common 50-day oscillation in atmospheric angular momentum and the length of day which was detected earlier in zonal wind anomalies of the tropical Pacific as 40-50 day oscillation by Madden and Julian [1972]. Djurović and Pâquet [1988] explained the origin of this 50-day oscillation in Earth rotation and atmospheric angular momentum by detection of such oscillation in other geophysical and solar activity phenomena such as geomagnetic field, sunspots, interplanetary magnetic field, and solar wind. Djurović and Pâquet [1996] also detected 5.5-year oscillation in the solar activity, Earth rotation, geomagnetic field and atmospheric circulations. The common short period oscillations with periods of 18 and 50 days with a good phase agreement and 27-day oscillation with a good amplitude agreement in Earth's rate of rotation, atmospheric angular momentum and geomagnetic index Ap were found by Kosek [1993]. The analysis of the amplitude of the semi-annual variation in LOD showed its strong modulation by the 11-year cycle which suggests that Sun can influence the zonal winds in the troposphere at the decadal to multi-decadal time scales [Le Mouël et al. 2010].

Solar irradiance is the Earth's primary energy input and external cause of terrestrial variability, which establishes the thermal and dynamical structure of the terrestrial environment. The TSI is known to be linked to the Earth climate and temperature variations. Total Solar Irradiance (TSI) is spatially and spectrally integrated radiant energy from the Sun as being measured at the top of the Earth's atmosphere and normalized to 1 AU [Kosek 1993]. TSI was accepted as constant until the satellite measurements since 1987 demonstrated its decadal variations related to the sunspot numbers variability [Hoyt and Schatten 1997; Parker 2000]. The historical TSI reconstructions for over the last 400 years are usually computed from the recent revisions of sunspotnumber records representing most reliable estimates of the solar variability [e.g. Hoyt and Shatten 1993]. Currently, 7 satellite independent measurement instruments are used in obtaining the TSI data, which makes it possible to ignore the impact of the Earth's atmosphere on the results [Scafetta and Willson 2014]. These measurements cover various periods, for example, the time series used in the study derived from Solar Radiation \& Climate Experiment Total Solar Irradiance Monitor (SORCE/TIM) cover the period from 2003 to now. The average value of the solar constant, measured by the SORCE experiment, is $1361 \mathrm{~W} / \mathrm{m}^{2}$ [Scafetta and Willson 2014].

The heating of Earth's surface and its fluid layers caused by TSI is not constant and even small changes in the solar output with the 11-year solar cycle could be amplified in these layers. Some recent papers have shown that the interannual and longer-period oscillations in the meteorological and climatic data are correlated with the solar activity [e.g. Hoyt and Schatten 1997; Lean and Rind 1999; Reid 1995; Sadourny 1994], and even small changes in the solar output could be amplified in the Earth's atmosphere [Arnold 1997; Schindell et al. 1999]. Soon [2005] showed that in 1875-2000 the changes in the annual arctic air temperature anomalies are correlated well with the TSI values reconstructed by Hoyt and Schatten [1993]. Soon and Legates [2013] found a correlation between the TSI and Equator-toPole temperature gradient trends. Moffa-Sanchez et al. [2014] found that the solar activity correlates well with North Atlantic temperatures. Soon et al. [2015] found a strong correlation between Northern Hemisphere (NH) temperatures and TSI datasets of Scafetta and Willson [2014] implying that the solar variability has been the dominant influence on NH temperature trends since at least 1881. Significant solar-related signals in the equatorial sea surface temperature (SST) over the entire 20th century were found by Lau and Weng [1995] and White et al. [1997]. Biktash [2019] found that the cyclical variations in the Earth's global temperature are related to the solar activity and are explained by the mechanism of the TSI effect on the Earth's atmosphere modulated by galactic cosmic rays. The impact of the TSI on LOD and UT1 centennial variations has been studied by Chapanov et al. [2017]. The solar origin of interannual oscillation from several months to 10 years in LOD, mean sea level and ENSO variability has been also studied by Chapanov et al. [2019] using the TSI and sunspot numbers data.

The aim of the study was to identify and compute oscillations in LODR and TSI time series with similar amplitude variations. 


\section{DATA SETS}

The LODR series (Fig.1) obtained from the IERS EOPC04R_IAU2000_daily data in the years 1962.1 to 2019.246 and the TSI series (Fig. 2) from the SORCE/TIM data in the years 2003.57 to 2019.246 were used in the analysis. The LODR data with one day sampling interval are available from the site (http://hpiers.obspm.fr/) and the TSI daily data are available from (http://lasp.colorado.edu/).

\section{ANALYSIS}

In order to compute the instantaneous amplitudes of oscillations, the combination of the Fourier transform band pass filter (FTBPF) with the Hilbert transform $(\mathrm{FTBPF}+\mathrm{HT})$ was used.

The wideband oscillation with central frequency $\omega_{o}$ computed by the FTBPF is given by the following formula [Kosek 1995]:

$$
x\left(t, \omega_{o}\right)=F T^{-1}\left[F T(x(t)) \cdot P\left(\omega, \omega_{o}\right)\right]
$$

where

$$
P\left(\omega, \omega_{o}\right)=\left\{\begin{array}{cc}
1-\frac{\left(\omega-\omega_{o}\right)^{2}}{\lambda^{2}}, & \left|\omega-\omega_{o}\right| \leq \lambda \\
0 & \text { otherwise }
\end{array}\right.
$$

is the parabolic transmittance function in which $\lambda$ is half of the filter bandwidth. The $\lambda>\pi / n$ parameter controls the time and frequency resolution of the filtered oscillation (eq. 1). The decrease/increase of $\lambda$ parameter makes this oscillation narrower/wider, respectively.
The instantaneous amplitude of the oscillation (eq. 1) is computed by the following formula:

$$
A\left(t, \omega_{o}\right)=\sqrt{\left[\mathfrak{R}\left(z\left(t, \omega_{o}\right)\right)\right]^{2}+\left[\Im\left(z\left(t, \omega_{o}\right)\right)\right]^{2}}
$$

where:

$$
\begin{gathered}
z\left(t, \omega_{o}\right)= \\
=F T^{-1}\left[F T(x(t)) P\left(\omega, \omega_{o}\right)(\operatorname{sign}(\omega)+1)\right]
\end{gathered}
$$

is the complex-valued oscillation computed by the FTBPF+HT [Popiński 2008; Gasquet and Witomski 1999; Kosek et al. 2016].

The spectra temporal correlation coefficient as a function of time $t$ and oscillation frequency $\omega_{o}$ between amplitude variations of oscillations in two real-valued $x(t)$ and $y(t)$ time series is given by the following formula:

$$
\begin{gathered}
C\left(t, \omega_{o}\right)=\frac{\sum_{k=-m}^{m}\left[\bar{A}_{x}\left(t+k, \omega_{o}\right)\left[\bar{A}_{y}\left(t+k, \omega_{o}\right)\right]\right.}{\sqrt{\sum_{k=-m}^{m}\left[\bar{A}_{x}\left(t+k, \omega_{o}\right)\right]^{2} \sum_{k=-m}^{m}\left[\bar{A}_{y}\left(t+k, \omega_{o}\right)\right]^{2}}} \\
m=\frac{6 \pi}{\omega_{o} \Delta t}, \\
\text { for } \quad t=n_{f}+m+1, m+2, \ldots, n-n_{f}-m,
\end{gathered}
$$

where

$$
\begin{aligned}
& \bar{A}_{x}\left(t, \omega_{o}\right)=A_{x}\left(t, \omega_{o}\right)-\frac{1}{n-2 n_{f}} \sum_{t=n_{f}}^{n-n_{f}} A_{x}\left(t, \omega_{o}\right) \\
& \bar{A}_{y}\left(t, \omega_{o}\right)=A_{y}\left(t, \omega_{o}\right)-\frac{1}{n-2 n_{f}} \sum_{t=n_{f}}^{n-n_{f}} A_{y}\left(t, \omega_{o}\right)
\end{aligned}
$$

are the residuals of oscillation amplitudes (eq. 3) in $x(t)$ and $y(t)$ time series, respectively, after removing their mean values, $n_{f}$ is the number of points to be dropped at the beginning and at the end of time series due to filter errors and $\Delta t$ is the sampling interval of data.

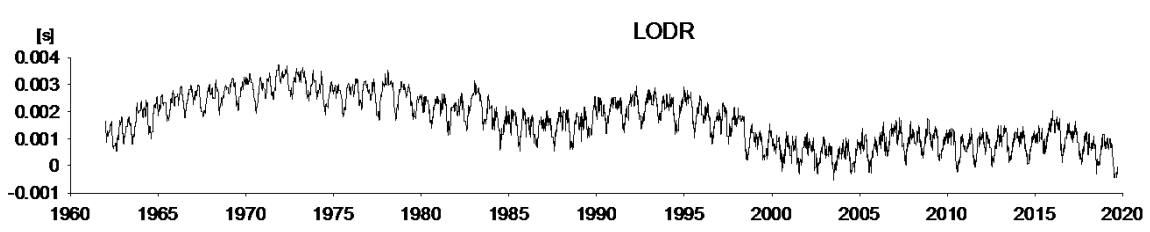

Figure 1. LODR daily time series data

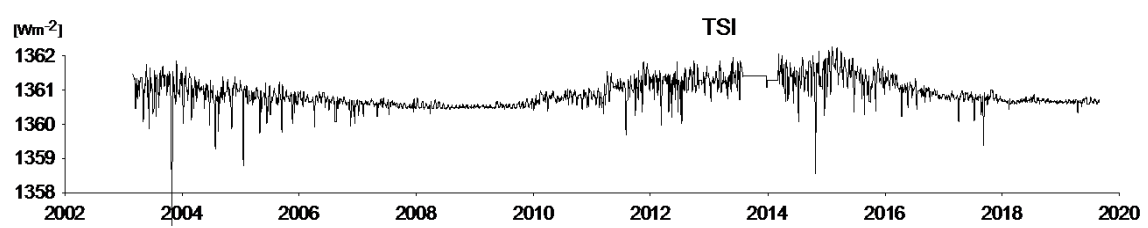

Figure 2. TSI daily time series data 
For particular value of $m=\frac{n-2 n_{f}}{2}+1$ (half of the total number of data $\eta$ the spectra-temporal correlation coefficient $C\left(t, \omega_{d}\right)$ becomes frequency dependent correlation coefficient $C\left(\omega_{\sigma}\right)$ between the amplitudes of oscillations. In these analyses $n_{f}=100$ was adopted for all considered oscillations.

\section{RESULTS}

The maps in figure 3 show spectra temporal correlation coefficients for two different $\lambda=0.001$ and $\lambda=0.002$ parameters between amplitude variations in LODR and TSI time series. It can be noticed that a decrease of the $\lambda$ parameter increase the frequency resolution, but at the same time the time resolution of information on the map decreases. High correlation coefficients occur for oscillation with periods slightly greater than 200 days and for the annual oscillation, but from 2007 to 2012 .

Figure 4 shows the frequency dependent correlation coefficients between the amplitude variations in LODR and the TSI time series computed for different $\lambda$ parameters $(0.001,0.0015$, 0.002 ). The maxima of these correlation coefficients detect oscillations with similar amplitude variations in the LODR and TSI time series. It can be noticed that these maxima may correspond to the oscillations with periods of 200-230 days, 100-120 days, $\sim 50$ and $\sim 30$ days.

Figure 5 shows the 32 days and 230 days oscillations in TSI and LODR with similar variations of amplitudes. It can be noticed that the phase of the oscillation with period of 230 days has several reverses (or jumps by 180 degree) in 2005, 2013 and 2015, and no agreement during 2007-2009.

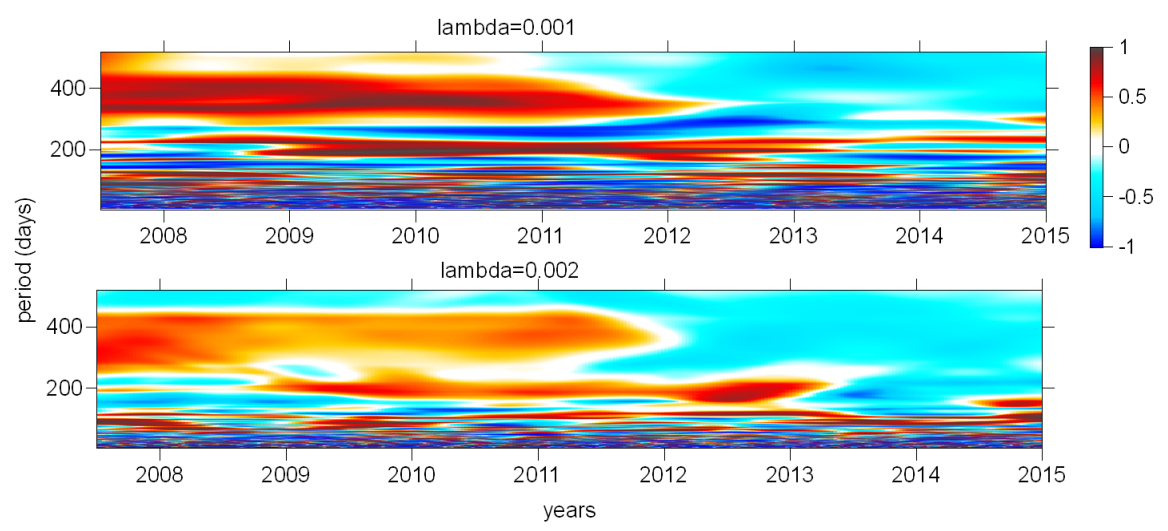

Figure 3. Spectra-temporal correlation coefficient between amplitude variations of oscillations in LODR and TSI time series

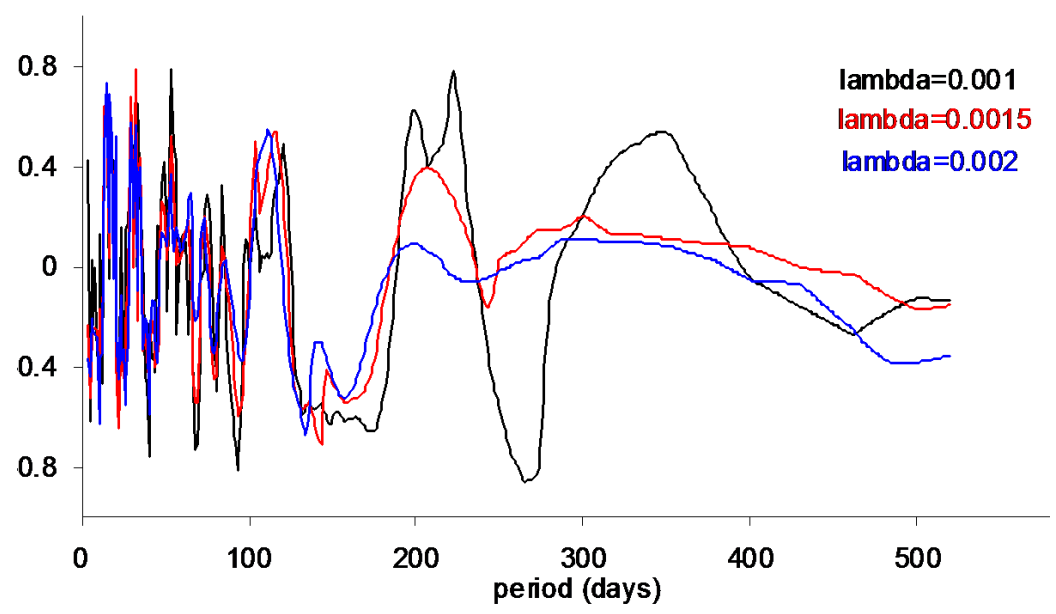

Figure 4. Frequency dependent correlation coefficient between amplitudes of oscillations in LODR and TSI computed for different $\lambda$ parameters $(0.001$ black, 0.0015 red and 0.002 blue) 
a)

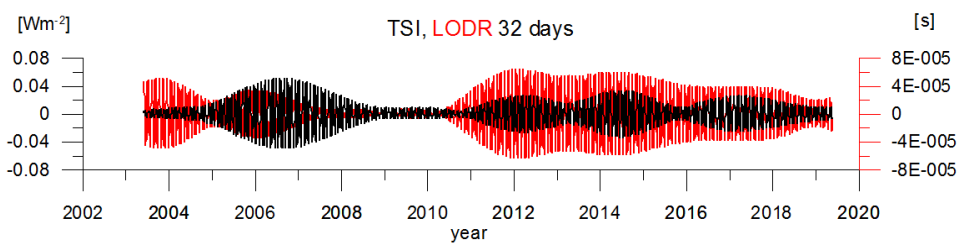

b)

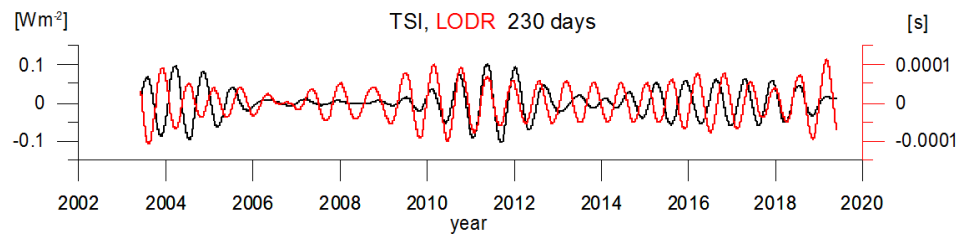

Figure 5. Oscillations in TSI (black) and LODR (red) time series with central periods of a) 32 days and b) 230 days computed by the FTBPF $(\lambda=0.001)$

\section{CONCLUSIONS}

1. The combination of the Fourier transform band pass filter and Hilbert transform allows detecting amplitude variations as a function of the oscillation period.

2. Amplitude variations in two different time series enable computation of frequency dependent or time-frequency correlation coefficients between them. It allows identifying such oscillations in two time series which have similar amplitude variations.

3. The method applied to the non-tidal length of day (LODR) data and the total solar irradiance (TSI) data etc, enable to detect a possible relationship between them.

4. This comparison method can be applied to any time series which consist of the oscillations with non-constant amplitudes.

\section{Acknowledgements}

Publication is funded by the Polish National Agency for Academic Exchange under the International Academic Partnerships Programme from the project ,Organization of the 9th International Scientific and Technical Conference entitled Environmental Engineering, Photogrammetry, Geoinformatics - Modern Technologies and Development Perspectives".

\section{REFERENCES}

1. Arnold, N.F., Robinson, T.R., 1997. Solar cycle changes to planetary wave propagation and their influence on the middle atmosphere circulation. Annales Geophysicae, 16(1), 69-76.

2. Biktash, L.Z., 2019. Influence of Total Solar
Irradiance on the Earth's Climate. Geomagnetism and Aeronomy, 59(3), 368-373.

3. Chapanov, Y., Ron, C., Vondràk, J., 2019. Solar origin of common interannual cycles of Earth rotation, MSL and climate. Fifteenth International Scientific Conference Space, Ecology, Safety 6-8 November 2019, Sofia, Bulgaria.

4. Chapanov, Y., Ron, C., Vondrak, J., 2017. Decadal cycles of Earth rotation, mean sea level and climate, excited by solar activity. Acta Geodyn. Geomater, 14, 241-250.

5. Chao, B.F., 1989. Length-of-day variations caused by El Nino-Southern Oscillation and quasi-biennial oscillation. Science, 243(4893), 923-925.

6. Dickey, J.O., Marcus, S.L., Hide, R., Eubanks, T.M., Boggs, D.H., 1994. Angular momentum exchange among the solid Earth, atmosphere, and oceans: A case study of the 1982-1983 El Niño event. Journal of Geophysical Research: Solid Earth, 99(B12), 23921-23937.

7. Dickey, J.O., Marcus, S.L., Hide, R., 1992. Global propagation of interannual fluctuations in atmospheric angular momentum. Nature, 357(6378), 484-488.

8. Djurovic, D., Pâquet, P., 1988. The solar origin of the 50-day fluctuation of the Earth rotation and atmospheric circulation. Astronomy and Astrophysics, 204, 306-312.

9. Djurović, D., Pâquet, P., 1996. The common oscillations of solar activity, the geomagnetic field, and the Earth's rotation. Solar Physics 167(1-2), 427-439.

10. Eubanks T.M., Steppe J.A., Dickey J.O., 1986. The El-Nino, the Southern Oscillation and the Earth Rotation. In: Cazenave A. (Ed.) Earth Rotation: Solved and Unsolved Problems. NATO ASI Series (Series C: Mathematical and Physical Sciences), Vol. 187, Springer, Dordrecht, 163-186.

11. Gambis, D. 2004. Monitoring Monitoring Earth orientation using space-geodetic techniques: stateof-the-art and prospective. Journal of Geodesy, 78(4-5), 295-303.

12. Gambis, D., 1992. Wavelet Transform Analysis of the Length of the Day and the El Nino/Southern Oscillation Variations at Intraseasonal and Interannual 
Time Scales, Ann. Geophys, 10, 429-437.

13. Gasquet, C., Witomski P., 1999. Fourier Analysis and Applications - Filtering, Numerical Computation, Wavelets, Springer Verlag Inc., New York.

14. Gross, R.S., 2007. Earth rotation variations - long period, in Physical Geodesy, edited by T.A. Herring, Treatise on Geophysics, Vol. 11, Elsevier, Amsterdam.

15. Haddad, M., Bonaduce, A., 2017. Interannual variations in length of day with respect to El Niño - Southern Oscillation's impact (1962-2015). Arab J Geosci, 10, 255.

16. Hoyt, D.V, Schatten, K.H., 1993. A discussion of plausible solar irradiance variations, 1700- 1992, J. Geophys. Res., 98(A11), 18,895-18,906.

17. Hoyt, D.V., Schatten, K.H., 1997. The Role of the Sun in Climate Change. Oxford University Press, New York.

18. Jault, D., Le Mouël, J.L. 1991. Exchange of angular momentum between the core and the mantle. Journal of geomagnetism and geoelectricity, 43(2), 111-129.

19. Kalarus, M., Schuh, H., Kosek, W., Akyilmaz, O., Bizouard, C., Gambis, D., Gross, R., Jovanović, B., Kumakshev, S., Kutterer, H., Mendes Cerveira, P. J., Pasynok, S., Zotov L., 2010. Achievements of the Earth orientation parameters prediction comparison campaign. Journal of Geodesy, 84(10), 587-596.

20. Kopp, G., 2016. Magnitudes and timescales of total solar irradiance variability. Journal of space weather and space climate, 6, A30.

21. Kosek, W., 1993. The common short periodic oscillations in the Earth's rate of rotation, atmospheric angular momentum and solar activity. Bulletin géodésique, 67(1), 1.

22. Kosek, W., 1995. Time variable band pass filter spectra of real and complex-valued polar motion series Time Artificial Satellites - Planetary Geodesy (no.24), 30(1), 27-43.

23. Kosek, W., Niedzielski T., Popiński W., ZbylutGórska M., Wnęk A. 2016. Variable seasonal and subseasonal oscillations in sea level anomaly data and their impact on prediction accuracy. In: International Association of Geodesy Symposia, Vol. 142, Springer, 1-6.

24. Langley, R.B., King, R.W., Shapiro, I.I., Rosen, R.D., Salstein, D.A., 1981. Atmospheric angular momentum and the length of day: A common fluctuation with a period near 50 days. Nature, 294(5843), 730-732.

25. Lau, K-M., Weng, H., 1995. Climate signal detection using wavelet transform: how to make a time series sing. Bull. Am. Meteor. Soc. 76, 2391-2402.

26. Le Mouël, J.-L., Blanter, E., Shnirman, M., and Courtillot, V., 2010. Solar forcing of the semiannual variation of length-of-day, Geophys. Res. Lett., 37, L15307.

27. Lean J., Rind, D., 1999. Evaluating sun-climate relationships since the little ice age. J. Atm. Terr. Phys., 61, 25-35.
28. Lei, Y., Zhao, D., Cai, H., 2015. Prediction of lengthof-day using extreme learning machine. Geodesy and geodynamics, 6(2), 151-159.

29. Liu, L.T., Hsu, H.T., Grafarend, E.W., 2005. Wavelet coherence analysis of length-of-day variations and El Nino-southern oscillation. Journal of Geodynamics, 39(3), 267-275.

30. Madden, R.A., Julian, P.R., 1972. Description of global-scale circulation cells in the tropics with a 40-50 day period. Journal of the atmospheric sciences, 29(6), 1109-1123.

31. Moffa-Sánchez, P., Born, A., Hall, I.R., Thornalley, D.J., \& Barker, S. 2014. Solar forcing of North Atlantic surface temperature and salinity over the past millennium. Nature geoscience, 7(4), 275-278.

32. Parker E.N. 2000. The physics of the sun and the gateway to the stars. Physics Today, 53, 26-31.

33. Popiński, W. 2008. Insight into the fourier transform band pass filtering technique. Artificial Satellites, 43(4), 129-141.

34. Ray R.D., Erofeeva, S.Y., 2014. Long-period tidal variations in the length of day. Journal of Geophysical Research: Solid Earth., 119 (2), 1498-1509.

35. Reid G.C. 1995. The sun-climate question: Is there a real connection? Rev. Geophys., 33, 535-538.

36. Sadourny C.R. 1994. L'influence du Soleil sur le climat. C.R. Acad. Sci. Paris, 319, 1325-1342.

37. Scafetta, N., Willson, R.C., 2014. ACRIM total solar irradiance satellite composite validation versus TSI proxy models. Astrophysics and Space Science, 350(2), 421-442.

38. Shindell, D.T., Rind, D., Balachandran, N., Lean, J., Lonergan, P., 1999. Solar cycle variability, ozone, and climate. Science, 284, 305-308.

39. Soon, W.W-H., Connolly, R., Connolly, M., 2015. Re-evaluating the role of solar variability on Northern Hemisphere temperature trends since the 19th century. Earth-Science Reviews, 150, 409-452.

40. Soon W.W-H., 2005. Variable solar irradiance as a plausible agent for multidecadal variations in the Arctic-wide surface air temperature record of the past 130 years, Geophysical Research Letters, Vol. 32, L16712.

41. Soon, W.W-H., Legates D., 2013. Solar irradiance modulation of Equator-to-Pole (Arctic) temperature gradients: Empirical evidence for climate variation on multi-decadal timescales. Journal of Atmospheric and Solar-Terrestrial Physics, 93, 45-56.

42. White, W.B., Lean, J., Cayan, D.R., Dettinger, M.D., 1997. Response of global upper ocean temperature to changing solar irradiance. J. Geophys. Res., 102, 3255-3266.

43. Yoder, C., Williams, J., Parke, J., 1981. Tidal variations of earth rotation. JGR, Vol, 86, Iss. B2, 881-891.

44. Zheng, D., Ding, X., Zhou, Y., Chen, Y., 2003. Earth rotation and ENSO events: combined excitation of interannual LOD variations by multiscale atmospheric oscillations. Global and Planetary Change, 36(1-2), 89-97. 